\title{
Dynamic expression analysis of armc10, the homologous gene of human GPRASP2, in zebrafish embryos
}

\author{
CHUNYU LIU $^{1 *}$, CHANGSONG LIN $^{1 *}$, JUN YAO $^{1}$, QINJUN WEI $^{1}$, GUANGQIAN XING $^{2}$ and XIN CAO ${ }^{1}$ \\ ${ }^{1}$ Department of Biotechnology, School of Basic Medicinal Sciences, Nanjing Medical University, Nanjing, Jiangsu 211166; \\ ${ }^{2}$ Department of Otolaryngology, The First Affiliated Hospital of Nanjing Medical University, \\ Nanjing, Jiangsu 210029, P.R. China
}

Received August 30, 2016; Accepted May 23, 2017

DOI: $10.3892 / \mathrm{mmr} .2017 .7357$

\begin{abstract}
G protein-coupled receptor-associated sorting protein 2 (GPRASP2), a member of the GASP family, has been reported to be involved in the modulation of transcription. However, few studies have revealed the role of GPRASP2 in the development and progression of diseases. As a model organism, zebrafish have been widely used to investigate human diseases. In the present study, zebrafish armadillo repeat-containing 10 (armc10), an orthologous gene of human GPRASP2 was identified, and the spatial and temporal expression patterns of armcl0 in zebrafish during early embryonic development were revealed. Bioinformatics analyses showed that ARMC10 protein sequences were highly conserved. Reverse transcription polymerase chain reaction analysis and whole mount in situ hybridization revealed that zebrafish armc10 was maternally expressed and was detected at a weak level up to $12 \mathrm{~h}$ post-fertilization (hpf), however, its expression increased to a high level at $24 \mathrm{hpf}$. At the $75 \%$ epiboly stage and $12 \mathrm{hpf}$, armcl0 was widely expressed in the embryo. At 24 hpf, armcl0 mRNA was expressed in the nervous system of the zebrafish head. When the embryo was 2 days old, the wide expression of armc10 was maintained in the nervous system of the zebrafish head. At $72 \mathrm{hpf}$, the mRNA expression of armc10 was located specifically in the otic vesicles in addition to the nervous system of the head. At $96 \mathrm{hpf}$, the expression of armcl0 was maintained in the otic vesicles and the nervous
\end{abstract}

Correspondence to: Professor Xin Cao, Department of Biotechnology, School of Basic Medicinal Sciences, Nanjing Medical University, 101 Longmian Road, Nanjing, Jiangsu 211166, P.R. China

E-mail: caoxin@njmu.edu.cn

Dr Guangqian Xing, Department of Otolaryngology, The First Affiliated Hospital of Nanjing Medical University, 300 Guangzhou Road, Nanjing, Jiangsu 210029, P.R. China

E-mail: xing-gq@163.com

*Contributed equally

Key words: zebrafish, armadillo repeat-containing 10, dynamic expression, G protein-coupled receptor-associated sorting protein 2 system of the head. The results of the present study provided novel insight into the spatial and temporal mRNA expression of armc10 in zebrafish, for the further investigation of nervous system diseases.

\section{Introduction}

G protein-coupled receptor (GPCR)-associated sorting protein 2 (GPRASP2), located at the chromosome region Xq22.1, is a member of the GPCR-associated sorting protein (GASP) family, comprising 10 members, which were identified by sequence homology searches $(1,2)$. It exhibits significant functions in modulating the activity of GPCRs (3), which triggers numerous cellular events, including the modification of secondary messenger levels (4), receptor desensitization and internalization (5), and modification of gene transcription $(6,7)$. For example, GASP-1 interacts with cytoplasmic tails of several GPCRs, including D2 dopamine receptor, $\delta$ opioid receptor $1, \beta-2$ adrenergic receptor and D4 dopamine receptor (8), and has been reported as an important breast cancer tumor and serum biomarker (9). GPRASP2 has been identified as a non-synonymous rare variant involved in the regulation of neurite outgrowth and other synaptic functions (10), and is an essential component of the Hedgehog-induced ciliary targeting complex, which regulates the translocation of Smoothened into the primary cilia (11). In addition, the knockdown of GPRASP2 has been shown to enhance hematopoietic stem cell repopulation (12). However, previous studies have shown that current understanding of the association between GPRASP2 and diseases remains limited.

Armadillo repeat-containing 10 (Armc10), a 343-amino acid protein, which contains six ARM repeats, is a member of the Armc10/Armadillo repeat-containing X-linked protein (Armcx) family of proteins, which exhibit a variety of functions in embryogenesis and tumorigenesis, including cell migration, cell proliferation, tissue maintenance, tumorigenesis, signal transduction and maintenance of overall cell structure $(13,14)$. The armcl0 gene is widely expressed in several species, and zebrafish armc10 has been found to be a homologous gene of GPRASP2 in our previous synteny analysis study (15).

To further examine the underlying molecular pathogenesis of GPRASP2, zebrafish at different embryonic stages were 
used in the present study as a model organism to perform whole mount in situ hybridization (WISH) and reverse transcription polymerase chain reaction (RT-PCR) analysis of zebrafish armc10, the homologous gene of human GPRASP2. The results revealed the spatial and temporal expression patterns of armcl0 in zebrafish during early embryonic development and assist in further understanding the role of GPRASP2 in embryogenesis and disease pathogenesis.

\section{Materials and methods}

Zebrafish care and maintenance. Zebrafish (Tübingen line) were provided by China Zebrafish Resource Center (Wuhan, China). The zebrafish care and experimental procedures were performed in accordance with the regulations set forth by the Institutional Animal Care and Use Committee of Nanjing Medical University (Nanjing, China). Zebrafish were maintained under $14 \mathrm{~h}$ light/10 h dark cycles and fed twice daily in a static water system at $28.5^{\circ} \mathrm{C}$. The vessels used for collecting embryos were placed at the four corners of the hydrostatic system fish tank 1 day prior to collecting embryos. The vessels were removed from the water following exposure to light for $30 \mathrm{~min}$ the subsequent day. The embryos were then raised at $28.5^{\circ} \mathrm{C}$ in an incubator following collection and washing. The embryonic stages were defined as described previously (16).

RNA purification and cDNA synthesis. Total RNA was extracted from 80 embryos at $24 \mathrm{~h}$ post-fertilization (hpf) using TRIzol reagent (Sangon Biotech Co., Ltd., Shanghai, China). Following extraction, $1 \mu \mathrm{g}$ of RNA was reverse transcribed into cDNA using RT Prime mix (Takara Bio, Inc., Otsu, Japan) according to the manufacturer's protocol. The primers were designed based on the sequences of armc10 (ENSDARG00000062960) provided by the Ensembl database (http://asia.ensembl.org/index.html) to clone the coding sequence of armc10. The primers used were as follows: armc10 F1, 5'-TGGGAGATGGCAGATGAT-3' and R1, 5'-AGGAGC CGTCCAGTAAAA-3'; armc10 F2, 5'-CTCTGCTGGGGA TTGTGG-3' and R2, 5'-GAGAGTCCGGTCTCCTCCTC-3'. The RT product was used as a template for nested-PCR with $10 \mu 1$ 2X PCR Mastermix (Beijing TransGen Biotech Co., Ltd., Beijing, China), $1 \mu \mathrm{l}$ cDNA, $2 \mu \mathrm{l} \mathrm{F/R}$ primers and $7 \mu \mathrm{l}$ $\mathrm{H}_{2} \mathrm{O}$. The conditions for the nested-PCR were as follows: $95^{\circ} \mathrm{C}$ for $3 \mathrm{~min}$, and 35 cycles of $95^{\circ} \mathrm{C}$ for $30 \mathrm{sec}, 56^{\circ} \mathrm{C}$ for $30 \mathrm{sec}$ and $72^{\circ} \mathrm{C}$ for $1 \mathrm{~min}$, followed by incubation for $10 \mathrm{~min}$ at $72^{\circ} \mathrm{C}$.

Probe synthesis. The cDNAs of the 3'untranslated region (3'UTR) of zebrafish armcl0 was used to amplify templates for the synthesis of armc10 antisense RNA probes using the following primer pair: F2-armc10-utr 5'-CTCTGCTGGGGA TTGT GG-3' and R2-armc10-utr 5'-GAGAGTCCGGTCTCC TCCTC-3'. The sequence was then cloned into the pGEM-T Easy vector with T7 and SP6 RNA polymerase promoter sequences for in vitro transcription.

The templates used for synthesizing armc10 antisense RNA probes were generated by PCR amplification using pGEMT-armc10 as templates. RNA probes were generated by in vitro transcription from the T7 RNA promoter, incorporating DIG-11-UTP (Roche Diagnostics, Indianapolis,
IN, USA) nucleotides, using Sp6 RNA polymerase with the MAXIscript kit (Ambion; Thermo Fisher Scientific, Inc., Waltham, MA, USA). The DNA template was removed from the synthesized probe by DNaseI treatment and the probe was purified using LiCl-based precipitation. The probe was dissolved in DEPC-treated water and stored at $-80^{\circ} \mathrm{C}$.

Sequence analysis. The full-length sequence of zebrafish was obtained from the GenBank database (https://www.ncbi.nlm .nih.gov/genbank/). The coding sequence data of zebrafish armc10 were analyzed using Jellyfish 1.1 (http://www .jellyfishsoftware.com/) (17). Multiple sequence alignment of the amino acid sequences was performed using ClustalX2 (http://www.clustal.org/) to identify the evolutionarily conserved regions of ARMC10 among animals. Mega 6.0 (http://www.megasoftware.net/) was used to construct a phylogenetic tree of the evolution of ARMC10. Synteny analysis was performed using the Ensembl database.

Detection of armc10 mRNA using RT-PCR analysis and WISH. The distribution of the mRNA expression of armcl0 was examined using RT-PCR analysis, as previously reported (18). The embryos were staged as previously described (16). The sequences of primers used to detect the presence of armcl0 cDNA during embryogenesis were armc10, F2 5'-CTCTGC TGGGGATTGTGG-3' and R2, 5'-GAGAGTCCGGTCTCC TCCTC-3'. PCR analysis was performed using, $10 \mu 1$ 2X PCR Mastermix (Beijing TransGen Biotech Co., Ltd.), $1 \mu \mathrm{l}$ cDNA, $2 \mu \mathrm{l} / \mathrm{R}$ primers and $7 \mu \mathrm{H}_{2} \mathrm{O}$ and the conditions were as follows: $95^{\circ} \mathrm{C}$ for $3 \mathrm{~min}$, and 35 cycles of $95^{\circ} \mathrm{C}$ for $30 \mathrm{sec}$, $56^{\circ} \mathrm{C}$ for $30 \mathrm{sec}$ and $72^{\circ} \mathrm{C}$ for $1 \mathrm{~min}$, followed by $10 \mathrm{~min}$ at $72^{\circ} \mathrm{C}$. The sensitivity of the RT-PCR analysis was controlled by performing amplification of zebrafish $\beta$-actin using the same cDNA as a template (19). The primers of $\beta$-actin were as follows: $\beta$-actin, forward 5'-CCAGACATCAGGGAGTGA-3' and reverse 5'-GATACCGCAAGATTCCATAC-3'.

WISH was performed as previously described $(16,17)$. To prevent the development of melanin pigmentation at later stages, $0.003 \%$ 1-phenyl-2-thiouera was added at $24 \mathrm{hpf}$. The concentration of the probe used in hybridization was $1.0 \mathrm{ng} / \mu \mathrm{l}$ for armc10. Images were captured using a stereoscopic microscope (Leica Microsystems GmbH, Wetzlar, Germany).

\section{Results}

Analysis of the zebrafish armclo gene. On examining the zebrafish genome (armc10; ensembl.org), it was found that the zebrafish armc10 gene (XM_009297973) is located on chromosome 25 , has six exons and encodes a 348 amino acid protein. The Armc10 protein contains a transmembrane domain at the N-terminus (aa7-29), a putative cleavage site (aa30-36) and a flanking basic region close to the transmembrane region, similar to that found in translocase of outer mitochondria membrane 20 and B-cell lymphoma 2, which predicts putative targeting to the outer mitochondrial membrane (20). Full-length Armc10 contains six Arm domains arranged in a DUF634 domain (aa 85-337), which are partially deleted in certain isoforms (21). Multiple sequence alignment of the amino acid sequences of ARMC10 derived from six different species shows a high level of conservation in the ARMC10 

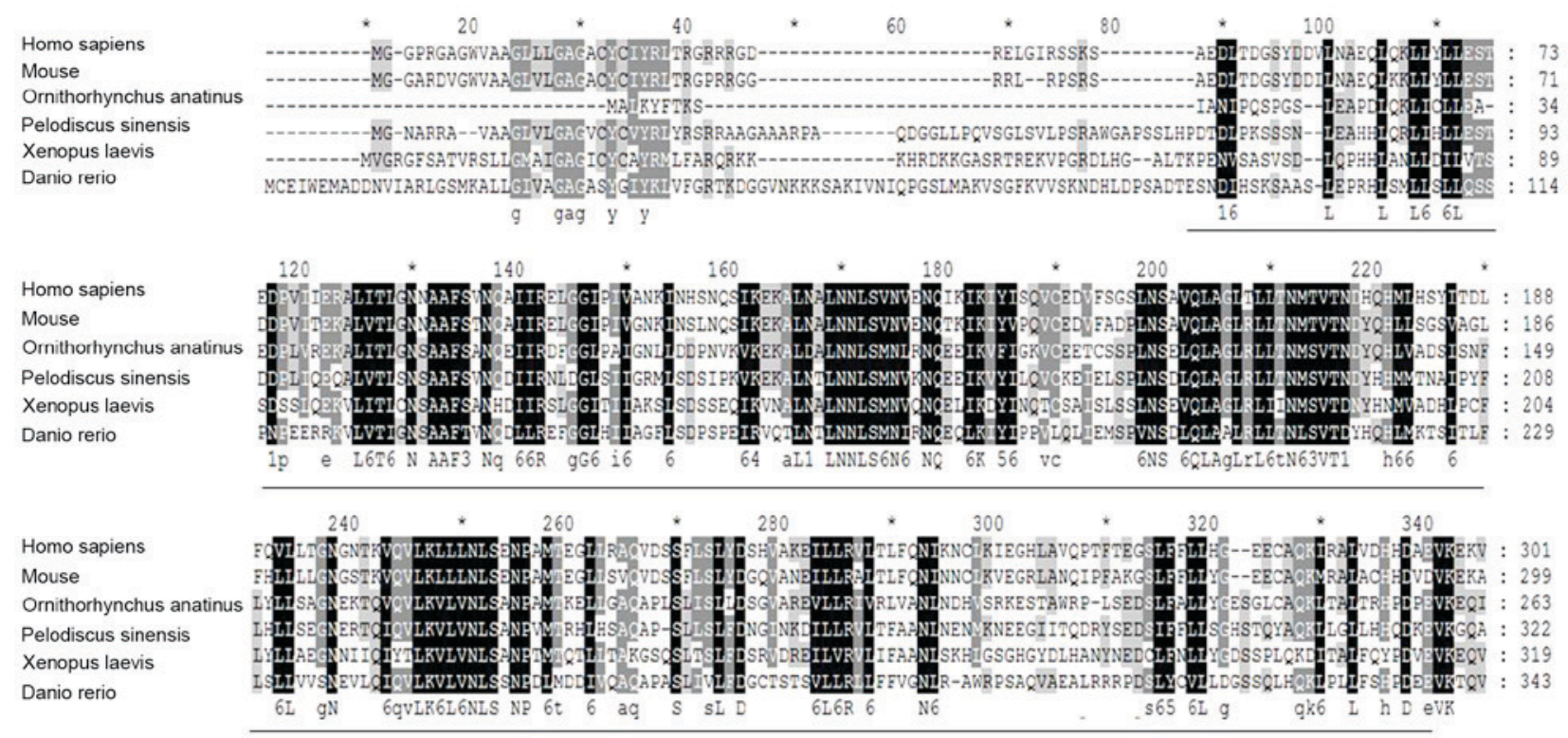

\begin{tabular}{|c|c|c|}
\hline & 360 & \\
\hline Homo sapiens & - & : 308 \\
\hline Mouse & ----- & : 306 \\
\hline Ornithorhynchus anatinus & 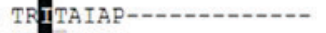 & : 271 \\
\hline Pelodiscus sinensis & AKIITLQRGDIQSPNPLLLFC & : 343 \\
\hline Xenopus laevis & LIINLARLRQL-------- & : 332 \\
\hline Danio rerio & 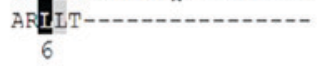 & : 348 \\
\hline
\end{tabular}

Figure 1. Amino acid sequence alignment of armc10 from several species. Sequences were aligned using ClustalX2. The armadillo domains are indicated by underlining. Conserved residues are shown in black ( $100 \%$ conservation), dark grey ( $80 \%$ conservation) and light grey (60\% conservation). An absence of shading denotes residues with $60 \%$ conservation. armc10, armadillo repeat containing 10 .

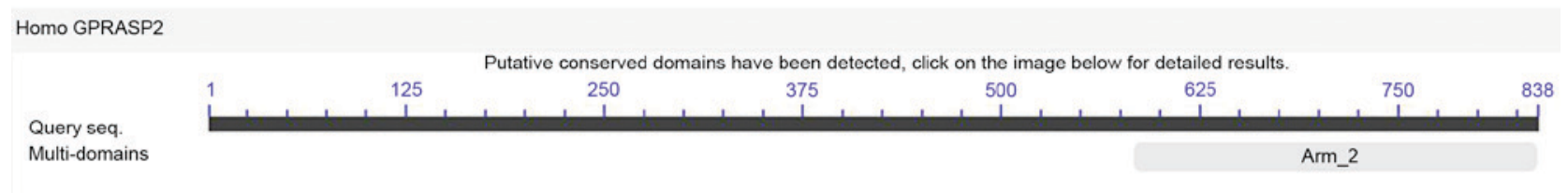

Zebrafish Armc10

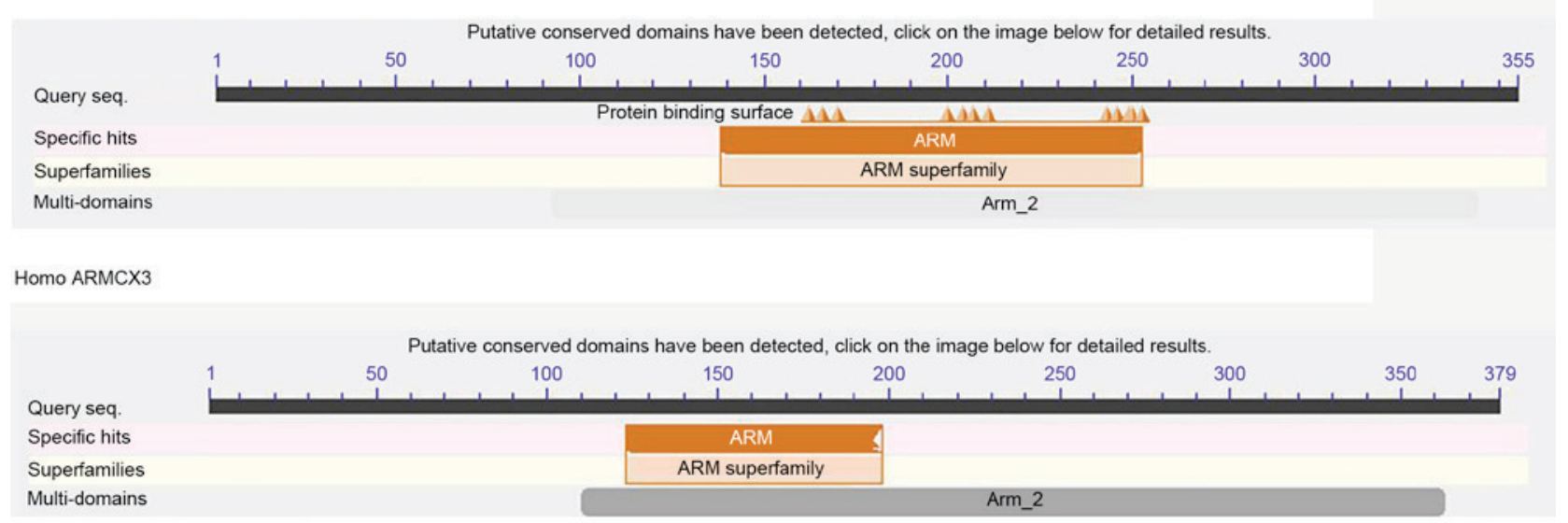

Figure 2. Analysis of conservative structure of protein functional domains. The ARM superfamily was present in the zebrafish Armc10 and Homo ARMCX3 sequences. Armc10, armadillo repeat-containing 10; ARMCX3, armadillo repeat-containing X-linked protein 3.

protein sequences among different species (Fig. 1). Typically, conserved Arm domains of 253 amino acids (22) were found to be distributed in ARMC10 (Fig. 1). The existence of ARM_2 multi-domains also confirmed the presence of the amino acid residues (Fig. 2).
Mega 6.0 was used to construct a phylogenetic tree of the evolution of ARMC10 using amino acid sequences from 32 species (Fig. 3). The results showed that sequences belonging to the same family or order were formed in a cluster. The zebrafish armc10 sequence formed one clad with that of 


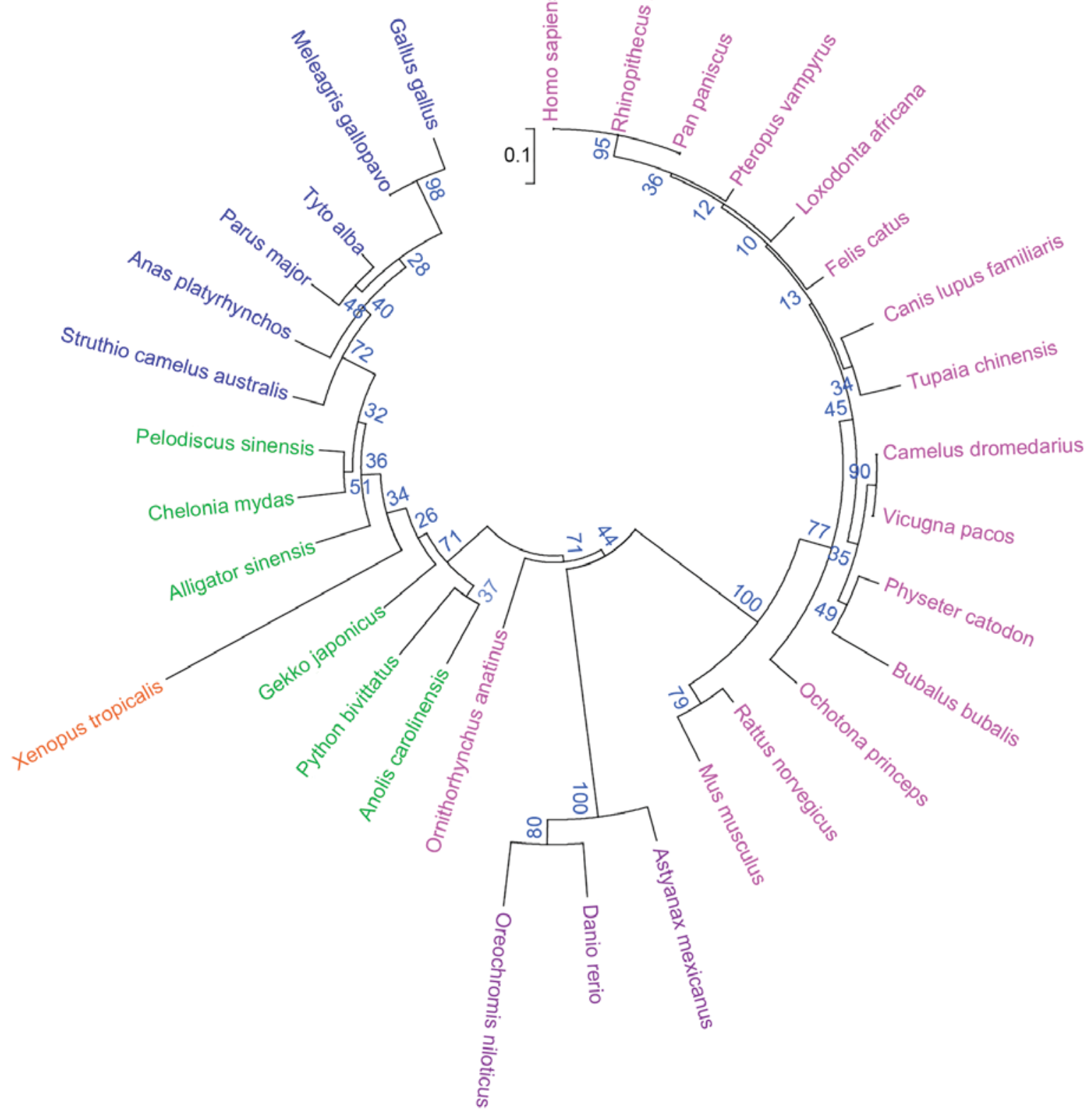

Figure 3. Phylogenetic tree of the zebrafish ARMC10 amino acid sequence with other species. A phylogenetic tree was constructed using the neighbor-joining method with Mega 6.0. Species belonging to same class were labeled in the same color: Pink, mammalia; violet, actinopterygii; green, reptilian; blue, aves; brown, amphibia. The ARMC10 sequences were retrieved from the GenBank database. ARMC10, armadillo repeat containing 10.

Oreochromis niloticus (bootstrap value 77). Higher bootstrap values were observed among the mammalians, including Rattus norvegicus, Ochotona princeps, Camelus dromedarius and Homo sapiens, Pan paniscus and Rhinopithecus roxellana). The Tyto alba, Parus major and Anas platyrhynchos species formed a clad, separating it from that of reptilia (Pelodiscus sinensis, Chelonia mydas and Alligator sinensis). The tree indicated that the ARMC10 protein underwent natural selection during evolution in accordance with the requirements of the environment.

Through blasting of the current zebrafish database in Ensemble with zebrafish armc10, the present study found that human GPRASP2 was a homologous gene of zebrafish armc10. Synteny analysis indicated that human GPRASP2
(NP_001171805) and human ARMCX3 (NP_775104) were paralogous genes with $12.2 \%$ identity (Fig. 4A), whereas human ARMCX3 (NP_775104) exhibited 25\% amino acid identity with zebrafish armc10 (Fig. 4B). Human GPRASP2 also shared $8.68 \%$ identity with zebrafish armcl0 (Fig. 4C). Therefore, human GPRASP2 and zebrafish armc10 were considered homologous genes.

Expression of armc10 during zebrafish embryonic development. To analyze the spatio-temporal expression patterns of armc10, the present study performed RT-PCR analysis and WISH at stages of zebrafish development from the cleavage stage until $96 \mathrm{hpf}$. The results of the RT-PCR analysis demonstrated that armc10 was expressed throughout 


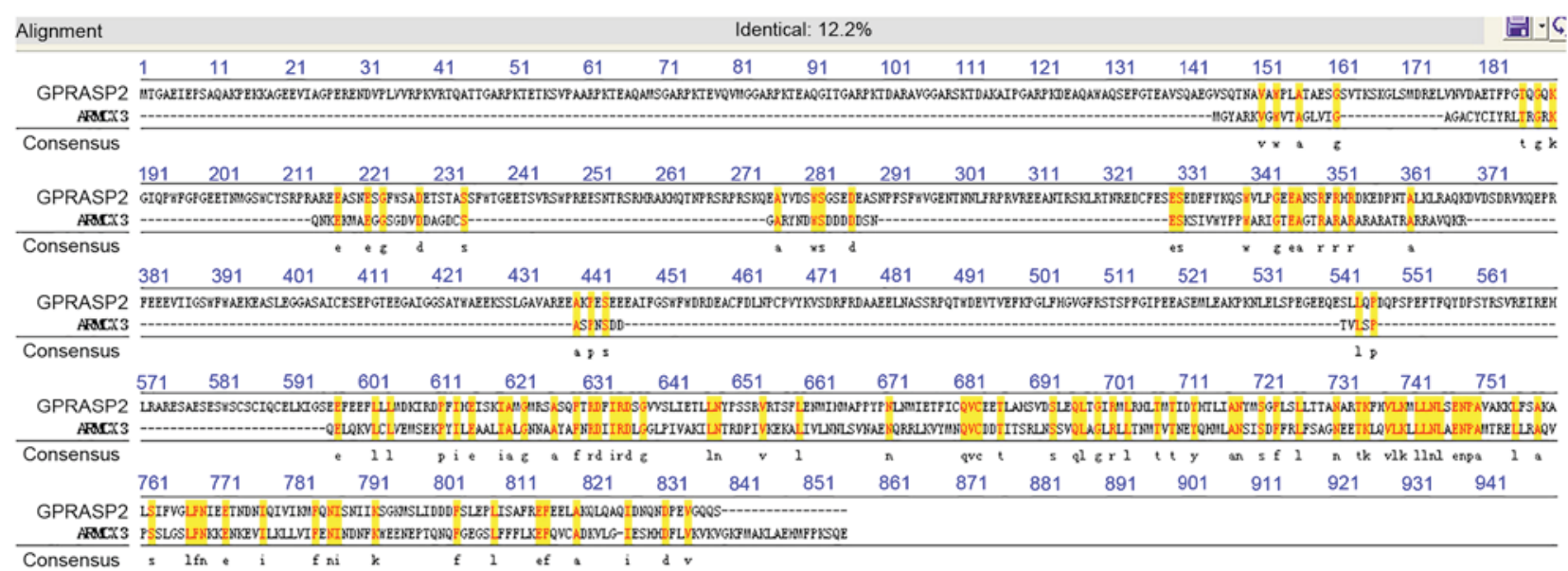

B

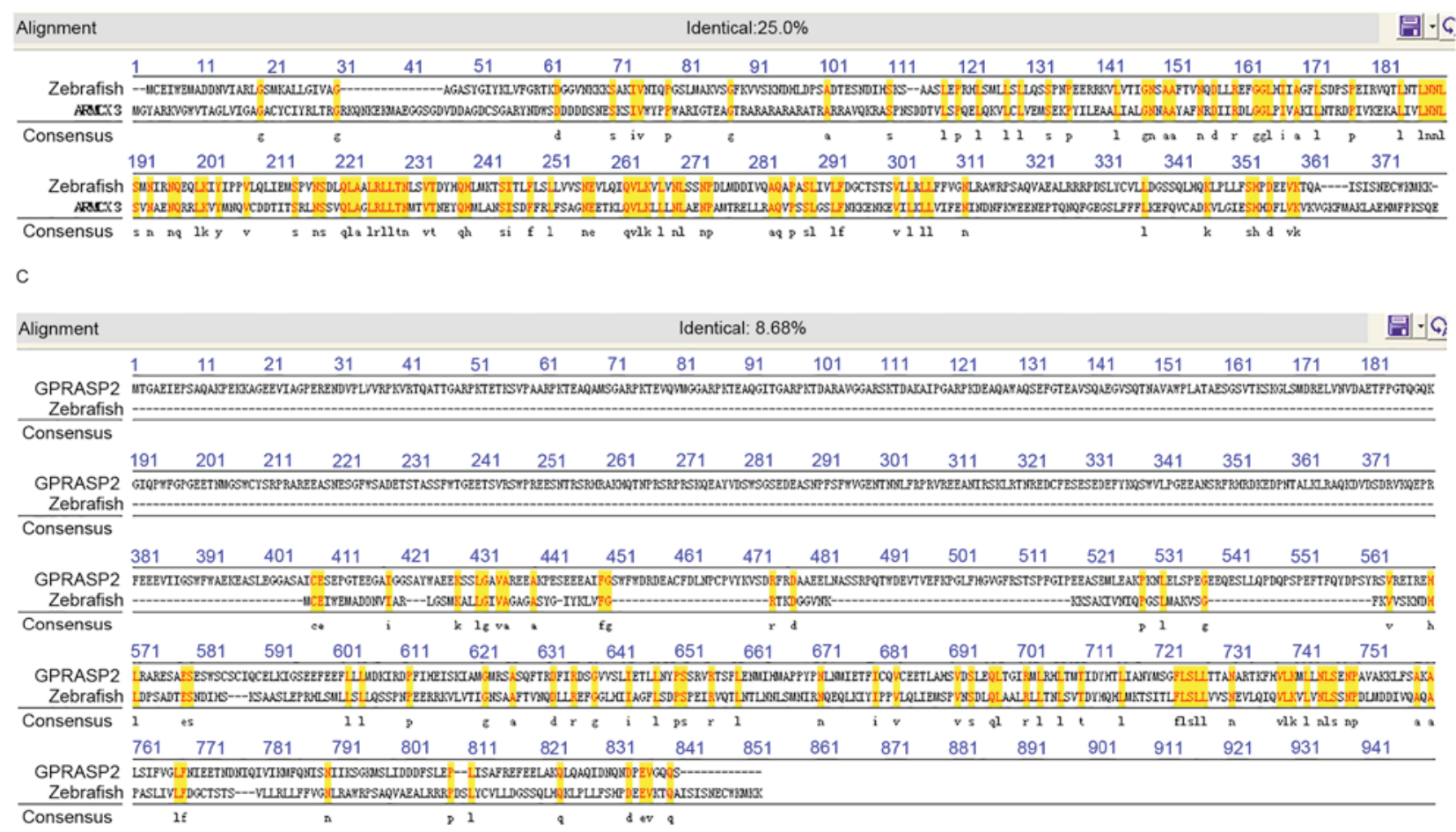

Figure 4. Amino acid sequence alignment of human GPRASP2 and other proteins. (A) Human GPRASP2 with human ARMCX3; (B) human ARMCX3 with zebrafish armc10; (C) human GPRASP2 with zebrafish Armc10. The same amino acids are marked in yellow. GPRASP2; G protein-coupled receptor-associated sorting protein 2; ARMCX3, armadillo repeat-containing X-linked protein 3; Armc10, armadillo repeat-containing 10.

early development. However, at the cleavage (two-cell) stage, $75 \%$ epiboly stage and at $12 \mathrm{hpf}$, the expression of armcl0 was weak. The embryos showed higher mRNA expression levels of armcl0 from $24 \mathrm{hpf}$ (Fig. 5). Consistent with the results of the RT-PCR analysis, WISH revealed that the hybridization signal of armcl0 was detected at the two-cell stage, indicating that armc10 was maternally expressed (Fig. 6A). At the 75\% epiboly stage and at $12 \mathrm{hpf}$, armc10 was widely expressed in the embryos (Fig. 6B and C). At $24 \mathrm{hpf}$, armc10 mRNA was expressed in the nervous system of the zebrafish head (Fig. 6D). When the embryos were 2 days old, armc10 maintained its wide expression in the nervous system of the zebrafish head (Fig. 6E). At $72 \mathrm{hpf}$, the armc10 mRNA was specifically expressed in otic vesicles in addition to the nervous system

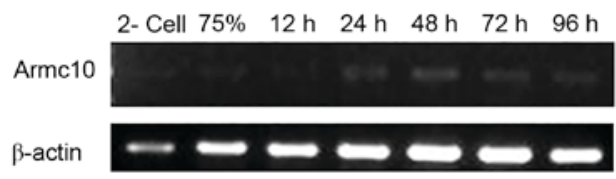

Figure 5. Temporal expression of zebrafish armc10. Temporal expression of zebrafish armc10 was analyzed using reverse transcription-polymerase chain reaction analysis. Zebrafish armc10 was first detected at the two-cell stage and persisted throughout development. $\beta$-actin was used as a control. armc10, armadillo repeat-containing 10 .

of the head (Fig. 6F). At $96 \mathrm{hpf}$, the expression of armcl0 remained in the otic vesicles and the nervous system of the head (Fig. 6G). 
A

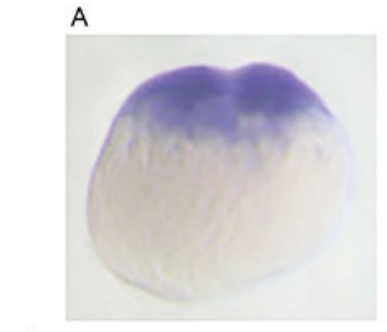

D

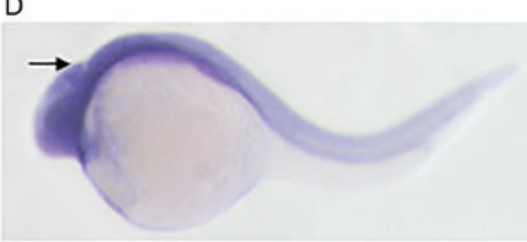

$\mathrm{F}$

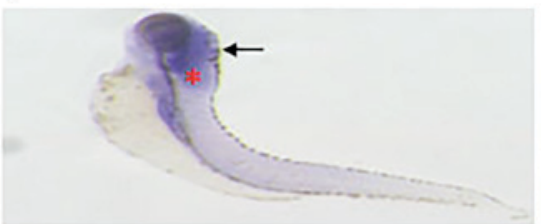

G

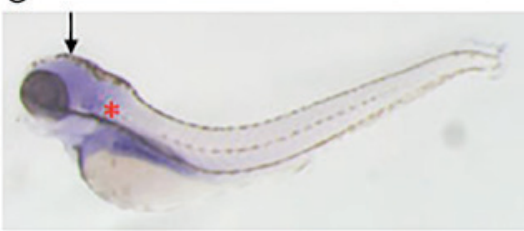

B

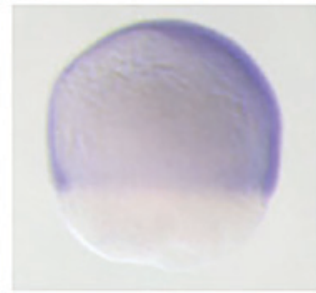

E
C

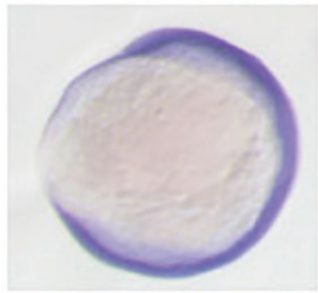

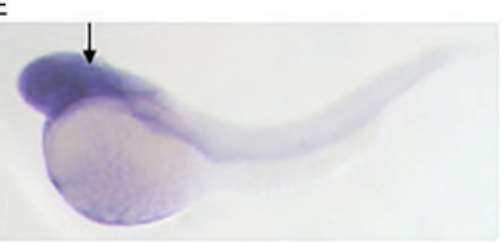
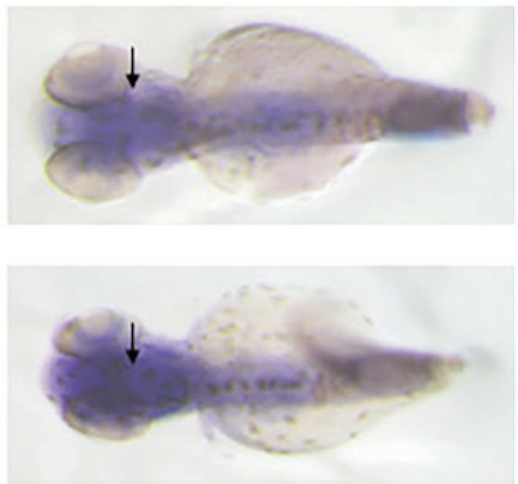

Figure 6. Expression of armc10 during early embryonic development of zebrafish. Dorsal views at (A) cleavage stage (two-cell), (B) 75\% epiboly, (C) 12 hpf, (D) $24 \mathrm{hpf}$, (E) $48 \mathrm{hpf}$. Views at (F) $72 \mathrm{hpf}$ (left, lateral view; right, dorsal view) and (G) $96 \mathrm{hpf}$ (left, lateral view; right, dorsal view). armc10, armadillo repeat-containing 10 .

\section{Discussion}

In the present study, to further examine the potential molecular pathogenesis of GPRASP2, the characterization and expression pattern of the homologous armcl0 gene in zebrafish were examined. The results of the bioinformatics analyses showed a high degree of conservation of the ARMC10 protein sequences among different species. The high degree of evolutionary conservation was particularly reflected by the presence of amino acid residues, which are important for protein-protein interactions, including the $\mathrm{N}$-terminus transmembrane domains and armadillo domains. The high conservation of the these domains is understandable, as it has been reported that the armadillo repeat domain is essential for protein-protein interactions (22-24) and is involved in diverse functions, including embryogenesis and tumorigenesis, by interacting with multiple binding partners (25). The phylogenetic analysis of ARMC10 using a phylogenetic tree demonstrated that the mammalian species formed a cluster with a higher bootstrap value and were closely associated with zebrafish, whereas variation was higher in lower organisms. It was concluded that ARMC10 gradually evolved from lower organisms with more variation, resulting in a more stable form in mammalian species. ARMC10 is also upregulated in hepatocellular carcinoma (26). Therefore, these results suggest a role for ARMC10 during embryogenesis and tumorigenesis.

In the present study, WISH and RT-PCR analysis were used to detect the expression of the zebrafish armclo gene during early embryogenesis. The results showed that armc10 was detected at low levels prior to $12 \mathrm{hpf}$, and the expression levels became higher at $24 \mathrm{hpf}$, distributed primarily in the regions of the nervous system and otic vesicles. These results were consistent to a previous finding that armc10 was widely expressed in adult nervous tissues, particularly in the forebrain regions of the cerebral cortex, hippocampus and thalamus (27). These sites of expression demonstrated that the expression of zebrafish armc10 was dynamic during embryogenesis. The spatial and temporal expression map of armc10, together with reports that the levels of armc10 regulate mitochondrial trafficking in neurons by controlling the number of moving mitochondria (21), suggest a role for armc10 in the pathophysiology of neurological diseases. Coincidentally, the syntenic analysis performed in the present study revealed that human GPRASP 2 and zebrafish armc10 were homologous genes. GPRASP2 has also been reported to be involved in receptor endocytosis and postsynaptic signaling via its interaction with the disease protein huntingtin, and that polyQ-dependent alterations of the interaction can contribute to the pathogenesis of Huntington's disease (28). Therefore, the conservation of protein sequences between zebrafish and higher vertebrates demonstrated in the present study using syntenic and homologous analysis suggested that investigations of zebrafish armcl0 may provide important insights into these processes in humans.

Taken together, the present study established the gene expression map of armcl0 among different stages of zebafish 
embryogenesis. The expression data compiled provided information relevant for future investigations of the role of armc10 in the nervous system during zebrafish embryogenesis, and provided information to assist in examining the mechanism of GPRASP2 associated with human nervous system diseases.

\section{Acknowledgements}

This study was supported by the grants from the National Natural Science Foundation of China (grant no. 31571302) and the Key Research and Development Program of Jiangsu Province (Social Development; grant no. BE2016762) to Professor Xin Cao; and grants from the Jiangsu Health Administration of China (grant no. LJ201120) and the Research Special Fund for Public Welfare Industry of Health, Ministry of Health of China (grant no. 201202005) to Dr Guangqian Xin.

\section{References}

1. Simonin F, Karcher P, Boeuf JJ, Matifas A and Kieffer BL: Identification of a novel family of $\mathrm{G}$ protein-coupled receptor associated sorting proteins. J Neurochem 89: 766-775, 2004.

2. Abu-Helo A and Simonin F: Identification and biological significance of $\mathrm{G}$ protein-coupled receptor associated sorting proteins (GASPs). Pharmacol Ther 126: 244-250, 2010.

3. Bornert O, Møller TC, Boeuf J, Candusso MP, Wagner R, Martinez KL and Simonin F: Identification of a nove protein-protein interaction motif mediating interaction of GPCR-associated sorting proteins with G protein-coupled receptors. PLoS One 8: e56336, 2013.

4. Gudermann T, Kalkbrenner F, Dippel E, Laugwitz KL and Schultz G: Specificity and complexity of receptor-G-protein interaction. Adv Second Messenger Phosphoprotein Res 31: 253-262, 1997.

5. Pierce KL, Premont RT and Lefkowitz RJ: Seven-transmembrane receptors. Nat Rev Mol Cell Biol 3: 639-650, 2002.

6. Pierce KL and Lefkowitz RJ: Classical and new roles of beta-arrestins in the regulation of G-protein-coupled receptors. Nat Rev Neurosci 2: 727-733, 2001.

7. West AE, Griffith EC and Greenberg ME: Regulation of transcription factors by neuronal activity. Nat Rev Neurosci 3 : 921-931, 2002.

8. Thompson D, Pusch M and Whistler JL: Changes in G protein-coupled receptor sorting protein affinity regulate postendocytic targeting of $\mathrm{G}$ protein-coupled receptors. J Biol Chem 282: 29178-29185, 2007.

9. Tuszynski GP, Rothman VL, Zheng X, Gutu M, Zhang X and Chang F: G-protein coupled receptor-associated sorting protein 1 (GASP-1), a potential biomarker in breast cancer. Exp Mol Pathol 91: 608-613, 2011.

10. Piton A, Gauthier J,Hamdan FF, Lafrenière RG, Yang Y, Henrion E, Laurent S, Noreau A, Thibodeau P, Karemera L, et al: Systematic resequencing of $\mathrm{X}$-chromosome synaptic genes in autism spectrum disorder and schizophrenia. Mol Psychiatry 16: 867-880, 2011.

11. Jung B, Padula D, Burtscher I, Landerer C, Lutter D, Theis F, Messias AC, Geerlof A, Sattler M, Kremmer E, et al: Pitchfork and gprasp2 target smoothened to the primary cilium for hedgehog pathway activation. PLoS One 11: e0149477, 2016.
12. Holmfeldt P, Ganuza M, Marathe H, He B, Hall T, Kang G, Moen J, Pardieck J, Saulsberry AC, Cico A, et al: Functional screen identifies regulators of murine hematopoietic stem cell repopulation. J Exp Med 213: 433-449, 2016.

13. Coates JC: Armadillo repeat proteins: Beyond the animal kingdom. Trends Cell Biol 13: 463-471, 2003.

14. Heydorn A, Søndergaard BP, Ersbøll B, Holst B, Nielsen FC, Haft CR, Whistler J and Schwartz TW: A library of 7TM receptor C-terminal tails. Interactions with the proposed post-endocytic sorting proteins ERM-binding phosphoprotein 50 (EBP50), N-ethylmaleimide-sensitive factor (NSF), sorting nexin 1 (SNX1), and G protein-coupled receptor-associated sorting protein (GASP). J Biol Chem 279: 54291-54303, 2004.

15. Xing G, Yao J, Liu C, Wei Q, Qian X, Wu L, Lu Y and Cao X: GPRASP2, a Novel causative gene implicated in an X-Linked recessive syndromic hearing loss. J Med Genet 54: 426-430, 2017.

16. Kimmel CB, Ballard WW, Kimmel SR, Ullmann B and Schilling TF: Stages of embryonic development of the zebrafish. Dev Dyn 203: 253-310, 1995.

17. Gu X, Xu F, Wang X, Gao X and Zhao Q: Molecular cloning and expression of a novel CYP26 gene (cyp26d1) during zebrafish early development. Gene Expr Patterns 5: 733-739, 2005.

18. Zhao Q, Dobbs-McAuliffe B and Linney E: Expression of cyp26b1 during zebrafish early development. Gene Expr Patterns 5: 363-369, 2005.

19. Sun L, Zou Z, Collodi P, Xu F, Xu X and Zhao Q: Identification and characterization of a second fibronectin gene in zebrafish. Matrix Biol 24: 69-77, 2005.

20. Rapaport D: Finding the right organelle. Targeting signals in mitochondrial outer-membrane proteins. EMBO Rep 4: 948-952, 2003.

21. Serrat R, Mirra S, Figueiro-Silva J, Navas-Pérez E, Quevedo M, López-Doménech G, Podlesniy P, Ulloa F, Garcia-Fernàndez J, Trullas R and Soriano E: The Armc10/SVH gene: Genome context, regulation of mitochondrial dynamics and protection against $A \beta$-induced mitochondrial fragmentation. Cell Death Dis 5: e1163, 2014.

22. McCrea PD, Turck CW and Gumbiner B: A homolog of the armadillo protein in Drosophila (plakoglobin) associated with E-cadherin. Science 254: 1359-1361, 1991.

23. Kawasaki Y, Senda T, Ishidate T, Koyama R, Morishita T, Iwayama Y, Higuchi $\mathrm{O}$ and Akiyama T: Asef, a link between the tumor suppressor APC and G-protein signaling. Science 289: 1194-1197, 2000

24. Eklof Spink K, Fridman SG and Weis WI: Molecular mechanisms of beta-catenin recognition by adenomatous polyposis coli revealed by the structure of an APC-beta-catenin complex. EMBO J 20: 6203-6212, 2001.

25. Hatzfeld M: The armadillo family of structural proteins. Int Rev Cytol 186: 179-224, 1999

26. Huang R, Xing Z, Luan Z, Wu T, Wu X and Hu G: A specific splicing variant of $\mathrm{SVH}$, a novel human armadillo repeat protein, is up-regulated in hepatocellular carcinomas. Cancer Res 63: 3775-3782, 2003.

27. López-Doménech G, Serrat R, Mirra S, D'Aniello S, Somorjai I, Abad A, Vitureira N, Garcia-Arumi E, Alonso MT, Rodriguez-Prados M, et al: The Eutherian Armcx genes regulate mitochondrial trafficking in neurons and interact with Miro and Trak2. Nat Commun 3: 814, 2012.

28. Horn SC, Lalowski M, Goehler H, Dröge A, Wanker EE and Stelzl U: Huntingtin interacts with the receptor sorting family protein GASP2. J Neural Transm (Vienna) 113: 1081-1090, 2006. 